\title{
Pandemics of Focal Plant Disease, a Model
}

\author{
F. van den Bosch, J. A. J. Metz, and J. C. Zadoks
}

First author: Mathematical Methods and Models, Section of Mathematics, Wageningen Agricultural University, Dreijenlaan 4, 6703 HA Wageningen, the Netherlands; second author: Institute of Evolutionary and Ecological Sciences, Theoretical Biology Section, Kaiserstraat 63, PO Box 9516, 2300 RA Leiden, the Netherlands, and Adaptive Dynamics Network, International Institute for Applied Systems Analysis, A-2361 Laxenburg, Austria; and third author: Department of Phytopathology, Wageningen Agricultural University, PO Box 8025,6700 EE Wageningen, the Netherlands.

Accepted for publication 22 January 1999

\begin{abstract}
van den Bosch, F., Metz, J. A. J., and Zadoks, J. C. 1999. Pandemics of focal plant disease, a model. Phytopathology 89:495-505.

An analytical model of a pandemic, initiated by a single focus and spreading over a continent, is developed using foci as the smallest units of disease and fields as the smallest units of host. A few generalizing assumptions lead to a parameter-sparse model that may answer general questions on pandemics in a qualitative manner. For pandemic spread of disease during one season, a 'within-season velocity of pandemic spread,' $C$, is expressed as a set of integral equations. Reduction of inoculum

pandemic spread over successive years is calculated as the 'polyetic velocity of pandemic spread,' $V$, which depends on $C$ and the push-back distance. The concept of 'pandemic effectiveness' is parameterized. Relations between the two velocities of pandemic spread and several model parameters are studied. Somewhat unexpectedly, velocities of pandemic spread depend only in a very limited way on field density represented by the 'cropping ratio' $\zeta$. This implies that our model and methods will also apply to situations with inhomogeneous field distributions. The effect of parameter values on rates of severity increase are analyzed. Finally, generalizations of the model are developed and their applications discussed.
\end{abstract} during the off-season is expressed by a 'survival ratio' of inoculum, $\varepsilon$. The effect of the off-season is a 'push-back' of the pandemic front over a distance $\Delta h$. It will be shown how $\Delta h$ is related to $C$ and $\varepsilon$. The mean
Additional keywords: disease spread, overseasoning, sanitation, seasonality.
An epidemic expanding over a continent is called a pandemic (9). A well-documented example is that of tobacco blue mold (Peronospora tabacina). The fungus, known from Australia in the 19th century (4), was reported in the United States in 1921. Between 1931 and 1950, a pandemic took place in North America. The velocity of pandemic spread was about $50 \mathrm{~km}$ per year (35). The disease appeared in England in 1959 and spread within 5 years over continental Europe and the Mediterranean countries (21) at a rate of about $130 \mathrm{~km}$ per year (35).

McGregor (15), discussing emigrant pests, stated that estimation of the relative danger posed by a quarantine pest and the design of emergency measures for containment and eradication would profit from models of pandemics with at least some predictive value (11). No model of pandemic spread has been formulated and analyzed to date.

The modeling of population expansion in space has a tradition in ecology and population genetics $(8,27)$. Kendall (12) was the first to model the spatial spread of diseases. A general model for the spatial expansion of age-structured populations $(5,6,28)$ was operationalized and applied to inter alia spatial expansions of vertebrate species on a continental scale $(16,30)$. Analytically tractable models were applied to the local expansion of a plant disease in onedimensional and two-dimensional plantings (17,18,29-33). Local epidemic spread was also described by means of spatially structured simulators $(35,38)$.

Here, we consider the step from small-scale spatial disease expansion to large-scale pandemics of focus-forming plant diseases. Focus formation is a short-time and small-scale phenomenon in

Corresponding author: F. van den Bosch

E-mail address: Frank.vandenbosch@ZTW.WK.WAU.NL

Publication no. P-1999-0419-01R

(C) 1999 The American Phytopathological Society which time and space are assumed to be continuous. For pandemic expansion, this assumption no longer holds. Epidemic progress within a field, consisting of both expansion of existing foci and initiation of new foci, is essentially different from the epidemic development in an assemblage of distant fields. Moreover, pandemics usually need several successive cropping seasons. We cannot ignore the discontinuity of time for pandemic expansion due to periods, often winters, in which disease development and spread is not possible. Furthermore, the discontinuity in space, a mixture of fields and nonfield areas, has to be incorporated in the model. In this paper, we combine the various ingredients to calculate the polyetic (36) or mean velocity during successive cropping seasons.

\section{MATERIALS AND METHODS}

Model. In this section, a model is derived for the spatial expansion of a focal plant disease. We limit the discussion to polycyclic (36) fungal disease propagated by airborne spores. We introduce some definitions to be used in the model derivation.

Continent. A pandemic spreads over a continent. A continent is considered to be an infinitely large, two-dimensional space. Position in space will be given in the usual Cartesian coordinate system $\left(x_{1}, x_{2}\right)$. We will often use a shorthand notation $\vec{x}=\left(x_{1}, x_{2}\right)$.

Crop. A crop is the agronomic entity grown continent-wide and susceptible to the disease under consideration. A crop occupies fields.

Field. A field is the geographical entity carrying a crop, with a position $\left(x_{1}, x_{2}\right)$. All fields are assumed to be equal in accessibility (36) to incoming spores, disease susceptibility, escapability of outgoing spores, climatic conditions, and cropping season.

Target area. The target area is the part of the continent covered by fields planted with the crop; the nontarget area is the part not covered by that crop. The ratio of target area to total area will be called the 'cropping ratio,' $\zeta$. Fields are assumed to be homogene- 
ously distributed over the continent. The assumption of homogeneity allows us to analytically calculate velocities and rates of disease increase. It will be shown that, due to the only slight dependence of velocities on the cropping ratio, inhomogeneous field distributions will not affect our results much (discussed below under Generalizations).

Season. The year consists of a cropping season and an off-season. The cropping season, 'season' for short, is the part of the year when fields are covered by the crop so that the epidemic can proceed. The off-season is the part of the year when there is no crop available.

Spatial spread within one cropping season. Individual. In modeling the dynamics of an infectious disease, one usually takes the (biological) individual as the conceptual unit. For plant disease, such an individual can be a fungal spore, a lesion, an infected leaf, or an infected plant. For modeling a pandemic, such a definition of individual is not suitable. The methods to calculate the velocity of spatial population expansion make use of the fact that density dependence in the far front of the epidemic is negligible $(5,30)$. In focal plant diseases, a nonnegligible depletion of susceptibles inside a focus will certainly occur, even in the far front

TABLE 1. Definitions of the variables, functions, and parameters used in the model

\begin{tabular}{|c|c|c|}
\hline & Definition & Dimension $^{\mathrm{a}}$ \\
\hline \multicolumn{3}{|l|}{ Variables } \\
\hline$\rho(t, \vec{x})$ & $\begin{array}{l}\text { Number of spores produced per unit of time by a } \\
\text { field at position } \vec{x}=\left(x_{1}, x_{2}\right) \text { at time } t \text { (field rate } \\
\text { of spore production) }\end{array}$ & $N_{\mathrm{s}} T^{-1} L^{-2}$ \\
\hline$b(t, \vec{x})$ & $\begin{array}{l}\text { Number of foci initiated per unit of time in a field } \\
\text { at position } \vec{x} \text { at time } t \text { (rate of focus initiation) }\end{array}$ & $N_{\mathrm{f}} T^{-1} L^{-2}$ \\
\hline$v(t, \vec{x})$ & $\begin{array}{l}\text { Number of between-field spores deposited per } \\
\text { unit of time at position } \vec{x} \text { and at time } t \text { (rate of } \\
\text { spore deposition) }\end{array}$ & $N_{\mathrm{s}} T^{-1} L^{-2}$ \\
\hline$N(t, \vec{x})$ & Number of foci in a field at position $\vec{x}$ at time $t$ & $N_{\mathrm{f}} L^{-2}$ \\
\hline \multicolumn{3}{|l|}{ Functions } \\
\hline & $\begin{array}{l}\text { Number of spores produced per unit of time by a } \\
\text { focus of age } a \text { (focus rate of spore production) }\end{array}$ & $N_{\mathrm{s}} T^{-1}$ \\
\hline$D(\vec{x}, \vec{\varphi})$ & $\begin{array}{l}\text { The probability density of a between-field spore } \\
\text { originating from a field at position } \vec{\varphi} \text { to be } \\
\text { deposited in a field at position } \vec{x} \text { (pandemic } \\
\text { dispersal distribution) }\end{array}$ & $L^{-2}$ \\
\hline$F(a)$ & $\begin{array}{l}\text { Number of foci initiated per unit of time due to } \\
\text { within-field and between-field spores produced } \\
\text { by a field infected time } a \text { ago }\end{array}$ & $N_{\mathrm{f}} T^{-1}$ \\
\hline \multicolumn{3}{|l|}{ Parameters } \\
\hline$a$ & Time since the initiation of a focus, age of a focus & $T$ \\
\hline$\alpha$ & Parameter in the spore production function $g(a)$ & $\begin{array}{l}N_{\mathrm{s}} T^{-2 \mathrm{~b}} \\
N_{\mathrm{s}} T^{-1 \mathrm{c}}\end{array}$ \\
\hline$\alpha \psi$ & Measure of pandemic effectiveness & $\begin{array}{l}N_{\mathrm{f}} T^{-2 \mathrm{~b}} \\
N_{\mathrm{f}} T^{-1 \mathrm{c}}\end{array}$ \\
\hline$C$ & Within-season velocity of pandemic spread & $L T^{-1}$ \\
\hline$\varepsilon$ & $\begin{array}{l}\text { Probability of a focus to survive the off-season } \\
\text { (survival ratio) }\end{array}$ & $N_{\mathrm{f}} N_{\mathrm{f}}^{-1}$ \\
\hline$\zeta$ & $\begin{array}{l}\text { Fraction of the continent covered by host fields } \\
\text { (cropping ratio) }\end{array}$ & 1 \\
\hline$\kappa$ & $\begin{array}{l}\text { Probability that a spore leaving the canopy is } \\
\text { redeposited in the field of its origin } \\
\text { (probability of within-field spore dispersal) }\end{array}$ & 1 \\
\hline$\lambda$ & Steepness parameter of the pandemic disease profile & $L^{-1}$ \\
\hline$\sigma$ & $\begin{array}{l}\text { Standard deviation of pandemic dispersal } \\
\quad \text { density, } D(\vec{x}, \vec{\varphi})\end{array}$ & $L$ \\
\hline$t$ & Time & $T$ \\
\hline$T$ & Duration of the crop season & $T$ \\
\hline$V$ & $\begin{array}{l}\text { The distance traveled during } 1 \text { year, polyetic } \\
\text { velocity of pandemic spread }\end{array}$ & $L$ \\
\hline$\vec{x}=\left(x_{1}, x_{2}\right)$ & Position on the two-dimensional continent & $L$ \\
\hline$\psi$ & $\begin{array}{l}\text { Probability that a spore landing in a field } \\
\text { initiates a new focus (success ratio) }\end{array}$ & $N_{\mathrm{f}} N_{\mathrm{s}}^{-1 \mathrm{~d}}$ \\
\hline
\end{tabular}

a $1=$ no dimension, $L=$ length, $N_{\mathrm{s}}=$ number of spores, $N_{\mathrm{f}}=$ number of foci, and $T=$ time.

${ }^{\mathrm{b}}$ According to Metz and van den Bosch (16).

c According to Minogue and Fry (17).

${ }^{\mathrm{d}} \psi$ has the dimension $N_{\mathrm{f}} N_{\mathrm{s}}^{-1}$ transforming number of spores into number of foci. of the epidemic. We circumvent this problem by taking the focus as our conceptual unit of modeling. The so-called 'linear conjecture' $(16,19,30)$ then allows us to neglect further density-dependent effects when calculating the rate of continental-scale disease spread.

Fate of a spore. We concentrate on diseases transmitted by airborne spores. Spores dispersed inside the canopy contribute to the development of the focus from which they originate, but they do not contribute to the initiation of new foci. We only consider spores that temporarily leave the canopy so that they can initiate new foci when they land elsewhere. Table 1 gives parameter definitions.

Consider a focus initiated time $a$ ago. We will loosely speak of $a$ as the 'age of the focus.' The number of spores leaving the canopy depends on focal age, $a$. By $g(a)$, we denote the number of spores (per unit of time) produced by a focus of age $a$ that leave the canopy. A spore leaving the canopy can be redeposited in the field where it originated with a probability, $\kappa$. These spores will loosely be called 'within-field spores.' With probability $1-\kappa$, a spore becomes subject to between-field dispersal so that it can initiate a new focus in another, maybe distant, field. Such spores are called 'between-field spores.' Thus, we distinguish within-field dynamics and between-field pandemic spread of the disease.

To describe the between-field dispersal of spores, we introduce the 'spore-dispersal density,' $D(\vec{x}, \vec{\varphi})$. This probability density is the probability that a spore originating from a source field at position $\vec{\varphi}$ is deposited in a target field at position $\vec{x}$. We assume that dispersal has no preferred direction and that dispersal is the same in every position on the continent. This implies that the dispersal probability only depends on the distance between the positions of source and target fields, $\vec{\varphi}$ and $\vec{x}$, respectively. Therefore, $D(\vec{x}, \vec{\varphi})=$ $D(|\vec{x}-\vec{\varphi}|)$, in which $|\vec{x}-\vec{\varphi}|=\sqrt{ }\left(x_{1}-\varphi_{1}\right)^{2}+\left(x_{2}-\varphi_{2}\right)^{2}$, is the distance between $\vec{x}$ and $\vec{\varphi}$.

Spore production. A field at position $\vec{x}$ and time $t$ can contain several foci of various ages. The total number of spores produced by the field per unit of time is denoted by $\rho\left(t, x_{1}, x_{2}\right)=\rho(t, \vec{x})$, which is the sum of within-field and between-field spores at $\vec{x}$ and $t$. Not every between-field spore will be deposited in a target field, since only a fraction, $\zeta$, of the continent is covered with host fields. The total number of spores dispersed between fields, originating from all possible fields on the continent, deposited per unit of time in a field at position $\vec{x}$ is denoted by $\mathrm{v}\left(t, x_{1}, x_{2}\right)=\mathrm{v}(t, \vec{x})$.

To calculate the total rate of spore production of a field, $\rho(t, \vec{x})$, we first consider foci of age $a$ in this field. The number of foci of age $a$ equals the number of foci initiated at time $t-a, b(t-a, \vec{x})$. These foci together produce $g(a) b(t-a, \vec{x})$ spores per unit of time. Foci of all possible ages contribute to the total rate of spore production of a field, $\rho(t, \vec{x})$. To calculate $\rho(t, \vec{x})$, we therefore must add all contributions of all focus ages to arrive at

$$
\rho(t, \vec{x})=\int_{0}^{t} b(t-a, \vec{x}) g(a) d a
$$

Spore deposition. Both between-field spores and within-field spores can initiate new foci in the crop at position $\vec{x}$. The probability that a spore landing in a field initiates a new focus is denoted by $\psi$. The number of new foci initiated per unit of time per unit of area in a field at position $\vec{x}$ at time $t$ is denoted by $b\left(t, x_{1}, x_{2}\right)=b(t, \vec{x})$. Note that $\mathrm{v}, b$, and $\rho$ are numbers per unit of time or rates per unit of area.

The total number of spores deposited per unit of time in a field at position $\vec{x}$ at time $t$ is the sum of within-field spores, $S_{1}$, and between-field spores, $S_{2}$. Given the probability of a spore deposited in a field to initiate a new focus, $\psi$, the number of foci initiated per unit of time, $b(t, \vec{x})$, equals

$$
b(t, \vec{x})=\psi\left(S_{1}+S_{2}\right)
$$

The parameter $\psi$ will be called the 'success ratio.' The deposition rate of within-field spores is $S_{1}=\kappa \rho(t, \vec{x})$, and the deposition rate of between-field dispersed spores is $S_{2}=v(t, \vec{x})$. 
Model completion. To complete the model, we derive a relation between the deposition rate of between-field spores in a field at $\vec{x}$ and the spore-production rate of fields all over the continent. Consider a field at position $\vec{\varphi}$. This field produces $(1-\kappa) \rho(t, \vec{\varphi})$ between-field spores per unit of time. The number of spores originating from $\vec{\varphi}$ and deposited at $\vec{x}$ equals $(1-\kappa) \rho(t, \vec{\varphi}) D(\vec{x}, \vec{\varphi})$. Multiplying this value by the cropping ratio, $\zeta$, yields the number of between-field spores originating from a source field at $\vec{\varphi}$ and deposited in target fields at position $\vec{x}$. To calculate the total number of spores deposited per unit of time in target fields at $\vec{x}$, $\mathrm{v}(t, \vec{x})$, we have to add all contributions of fields at all possible places $\vec{\varphi}$. The resulting expression for $v(t, \vec{x})$ is

$$
v(t, \vec{x})=\zeta(1-\kappa) \int_{-\infty}^{\infty} \int_{-\infty}^{\infty} \rho(t, \vec{\varphi}) D(|\vec{x}-\vec{\varphi}|) d \varphi_{1} d \varphi_{2}
$$

Putting all pieces together and substituting equation 1 where appropriate, the model finally reads

$$
\begin{aligned}
& b(t, \vec{x})=\psi \kappa \int_{0}^{t} b(t-a, \vec{x}) g(a) d a+\psi v(t, \vec{x}) \\
& v(t, \vec{x})=\zeta(1-\kappa) \int_{0}^{t} \int_{-\infty}^{\infty} \int_{-\infty}^{\infty} b(t-a, \vec{\varphi}) g(a) D(|\vec{x}-\vec{\varphi}|) d \varphi_{1} d \varphi_{2} d a
\end{aligned}
$$

If one is not interested in the rate at which new foci are initiated in a field at position $\vec{x}$ but in the number of foci in this field, $N(t, \vec{x})$, this can be calculated from

$$
N(t, \vec{x})=\int_{0}^{t} b(t-a, \vec{x}) d a
$$

Reformulation of the model. Appendix I shows that the model can be rewritten as

$$
b(t, \vec{x})=\int_{0}^{t} \int_{-\infty}^{\infty} \int_{-\infty}^{\infty} b(t-a, \vec{\varphi}) F(a) D(|\vec{x}-\vec{\varphi}|) d \varphi_{1} d \varphi_{2} d a
$$

in which

$$
F(a)=\zeta \frac{1-\kappa}{\kappa} \sum_{i=1}^{\infty}(\kappa \psi)^{i} g^{*_{i}}(a)
$$

In equation 6 , the term $g^{* i}(a)$ is the so-called convolution of $i$ times the function $g(a)$, defined as

$$
g^{*_{i}}(a)=\int_{0}^{a} g(\tau) g^{*(i-1)}(a-\tau) d \tau
$$

for $i=2,3, \ldots$ and $g(a)^{* 1}=g(a)$.

Velocity of pandemic spread. The model in its reformulated form (equation 5) is formally equivalent to a general model used to study the spread of animal, plant, and pathogen populations. This model and its nonlinear variants have been studied extensively $(5,6,16,19,28-33)$. Here, we state the main results to be used in this paper.

Since the between-field dispersal density is rotationally symmetric, the pandemic has circular symmetry also. Therefore, the following can be limited to a transect along the $x_{1}$ axis. When a new disease is locally introduced into a continent, the epidemic will spread over the continent as a traveling wave (37). Such a traveling wave can be visualized as a disease profile, with a fixed shape in space. This disease profile moves through space at a constant velocity. The disease profile of the traveling-wave solution of equation 5 has an exponential shape given by

$$
b\left(t, x_{1}, 0\right)=M e^{\lambda\left(c t-x_{1}\right)}
$$

in which $C$ is the velocity of the traveling wave, $\lambda$ is the steepness parameter of the disease profile, and $M$ is a parameter (Fig. 1A).
The results reported in the literature on equation 5 imply that the velocity, $C$, and steepness, $\lambda$, of the (only relevant) travelingwave solution can be calculated from

$$
\left\{\begin{array}{c}
L(C, \lambda)=1 \\
\frac{\partial L(C, \lambda)}{\partial \lambda}=0
\end{array}\right.
$$

in which

$$
L(C, \lambda)=\int_{0}^{\infty} e^{-\lambda C a} F(a) d a \int_{-\infty}^{\infty} e^{\lambda x_{1}} \widetilde{D}\left(x_{1}\right) d x_{1}
$$

is the characteristic equation and

$$
\widetilde{D}\left(x_{1}\right)=\int_{-\infty}^{\infty} D\left(x_{1}, x_{2}\right) d x_{2}
$$

is the marginal distribution of the spore-dispersal density, $D(\vec{x})$. Calculation of the within-season velocity of pandemic spread, $C$, is only possible if the integrals in equation 10a exist. This condition does not cause problems with the functions $F(a)$ and $g(a)$, but it imposes a biologically relevant restriction on the second integral of equation 10a, which only exists when the tail of $D(\vec{x})$ falls off exponentially or steeper with increasing $\vec{x}$. Severity dependence in the rate of a field's spore release and severity dependence in the probability that a deposited spore initiates a new focus has been neglected in the current model. However, this will not affect our results (discussed below under Generalizations).

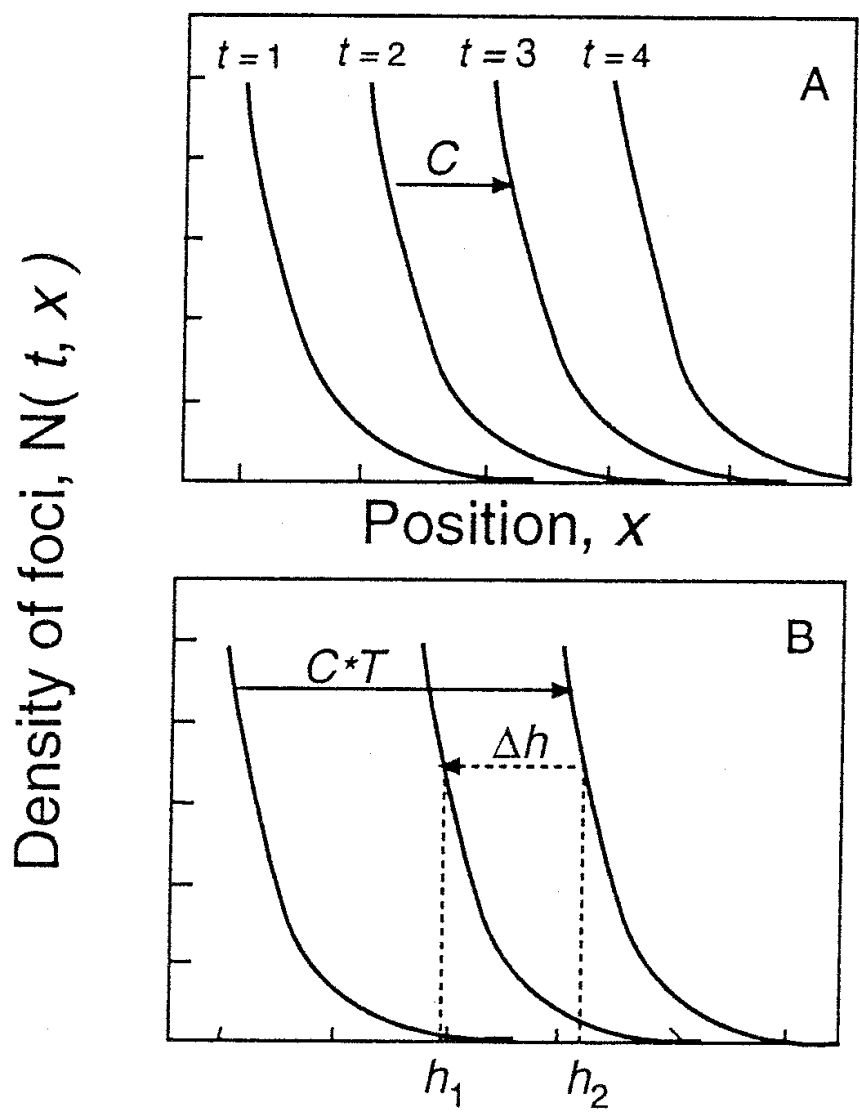

Fig. 1. Number of foci, $N(t, \vec{x})$, as function of position. A, The pandemic disease profile for various times, $t$, during one cropping season. $C$ is the within-season velocity of pandemic spread. B, The development of the pandemic profile during 1 year. Left-most curve $=$ pandemic disease profile at the onset of the cropping season. Right-most curve $=$ pandemic disease profile at the end of the cropping season (the start of the off-season). Middle curve $=$ pandemic disease profile at the end of the off-season. $C^{*} T=$ the total distance traveled by the pandemic wave during one cropping season. The left-pointing dotted arrow indicates the push-back distance, $\Delta h$. 
Measures of severity increase. Equation 8 and Figure 1 make clear that a traveling-wave solution has two relevant measures of severity increase. First, the steepness of the pandemic profile, $\lambda$, is a measure of severity increase in the spatial dimension at any time. This measure of severity increase can be seen as relevant for a distant observer. Second, the velocity $C$ multiplied by the steepness parameter $\lambda$ is the rate of severity increase in a field at position $\vec{x}$. This measure of severity increase is relevant to a local observer. These two measures of severity increase and the velocity of pandemic spread will be studied in the next sections.

Spread during successive cropping seasons. Model equations 4 describe the epidemic expansion within one cropping season. The duration of a cropping season will be denoted by $T$. During one cropping season the epidemic wave travels a distance $C T$. Considering a transect along the $x_{1}$ axis, the pandemic disease profile at the end of the cropping season at time $t=T$ is

$$
N\left(T, x_{1}, 0\right)=N e^{\lambda\left(C T-x_{1}\right)}
$$

in which $N$ is the number of foci in a field at position $\vec{x}$. At the end of the cropping season, $t=T$, the crop is harvested and most inoculum is removed from the field. The remaining inoculum has to survive the off-season on plant parts not removed during harvest; on cull piles, as for Phytophthora infestans; or on secondary hosts. Only a fraction of the inoculum will survive the off-season. At the start of the next cropping season, the surviving inoculum can induce one or more new foci. We will denote by $\varepsilon$ the number of foci at the beginning of the new cropping season relative to the number of foci at the end of the preceding cropping season. We will loosely speak of $\varepsilon$ as the 'survival ratio' of foci. The disease profile at the start of the next cropping season equals disease profile (equation 11) multiplied by $\varepsilon$,

$$
N\left(T, x_{1}, 0\right)=\varepsilon N e^{\lambda\left(C T-x_{1}\right)}
$$

Equation 12 implies that the steepness of the pandemic disease profile has not changed during the off-season. The effect of the off-season is only to push back the disease profile over a certain distance (Fig. 1B, $\Delta h$ ). To calculate this distance, consider a position $h_{1}$, in which at the end of the cropping season the number of foci in a field is $\theta$. At the start of the next cropping season, this same disease level $\theta$ now has position $h_{2}$. Substituting in equations 11 and 12, we find

$$
N e^{\lambda\left(C T-h_{1}\right)}=\varepsilon N e^{\lambda\left(C T-h_{2}\right)}
$$

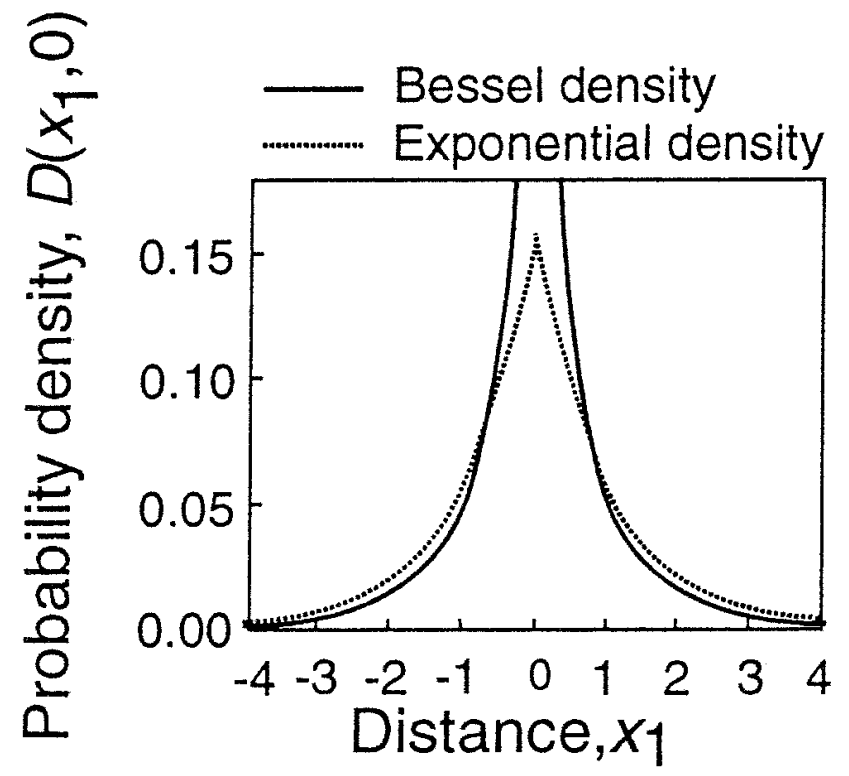

Fig. 2. Transect of the between-field-dispersal densities used as special cases of the general model. and the push-back distance $\Delta h$ is

$$
\Delta h=h_{1}-h_{2}=\frac{1}{\lambda} \ln \left(\frac{1}{\varepsilon}\right)
$$

The mean velocity of pandemic spread over a continent during successive cropping seasons, $V$, here called the 'polyetic velocity of pandemic spread,' is the distance traveled during a cropping season, $C T$, minus the push-back distance. Therefore, $V$ is calculated from

$$
V=C T-\frac{1}{\lambda} \ln \left(\frac{1}{\varepsilon}\right)
$$

in which $C$ and $\lambda$ are calculated from equations 9 and 10 .

Submodels. To actually calculate the velocity of pandemic spread from model equations 4 using equations 9 and 10, the functions $g(a)$ and $D(\vec{x})$ have to be specified. In this section, we introduce simple parameter-sparse submodels for these two functions. By comparing velocities of pandemic spread for these submodels, we get insight in the influence of the details of the spore production, $g(a)$, and spore dispersal, $D(\vec{x})$, functions on the velocity of pandemic spread.

TABLE 2. The equations for the within-season velocity of pandemic spread, $C$, and the steepness parameter of the pandemic disease profile, $\lambda^{\mathrm{a}, \mathrm{b}, \mathrm{c}}$

\begin{tabular}{lcc}
\hline$D(\vec{x}) g(a)$ & Bessel & Exponential \\
\hline Linear & Solve $Z$ numerically from \\
$Z^{3}-3 Z^{2}+Z\left(3-\frac{27}{4} A^{2}\right)-1=0$ \\
$C=\sqrt{\alpha \psi} \sigma \frac{1}{\sqrt{2}} \sqrt{\kappa} \sqrt{\frac{1+A+\sqrt{A^{2}+A}}{1+A-\sqrt{A^{2}+A}}}$ & Now $C=\sqrt{\alpha \psi} \sigma \sqrt{\kappa Z}$ \\
$\lambda=\frac{1}{\sigma} \sqrt{2} \sqrt{1+A-\sqrt{A^{2}+A}}$ & and $\lambda=\frac{1}{\sigma} \sqrt{\frac{1+2 Z}{3 Z}}$
\end{tabular}

Constant Solve $Z$ numerically from $Z^{3}+2 A Z^{2}-A=0$

$$
\begin{array}{cc}
C=\alpha \psi \sigma \frac{1}{\sqrt{2}} \kappa\left(1+\frac{3}{4} A+\frac{1}{4} \sqrt{9 A^{2}+8 A}\right) B & \text { Now } C=\alpha \psi \kappa \sigma \frac{A+Z}{Z \sqrt{1-Z^{2}}} \\
\text { in which } B=\sqrt{\frac{3 A+\sqrt{9 A^{2}+8 A}}{-A+\sqrt{9 A^{2}+8 A}}} & \text { and } \\
\lambda=\frac{\sqrt{2}}{\sigma} \sqrt{1-\frac{4 A}{3 A+\sqrt{9 A^{2}+8 A}}} & \lambda=\frac{1}{\sigma} \sqrt{1-Z^{2}}
\end{array}
$$

a In which $A=\zeta \frac{1-\kappa}{\kappa}$.

${ }^{\mathrm{b}}$ For $\kappa=0$ the following formulas apply:

Bessel + Linear $C=2 \sigma \sqrt{\alpha \psi \zeta}$

Bessel + Constant $C=\frac{3 \sqrt{3}}{2} \sigma \alpha \psi \zeta$

Exponential + Linear $C=\sqrt{\frac{3 \sqrt{3}}{2}} \sigma \sqrt{\alpha \psi \zeta}$

Exponential + Constant $C=2 \sigma \alpha \psi \zeta$.

${ }^{\mathrm{c}}$ For $\zeta$ approaching 0 the following formulas apply:

Bessel + Linear $C=\sigma \sqrt{\frac{1}{2} \alpha \psi \kappa}$

Bessel + Constant $C=\sigma \sqrt{1 / 2} \alpha \psi \kappa$

Exponential + Linear $C=\sigma \sqrt{\alpha \psi \kappa}$

Exponential + Constant $C=\sigma \alpha \psi \kappa$. 
Spore-production function. After a build-up phase, a focus expands radially at constant velocity $(30,32)$. Since the amount of host material is limited, a burnt-out zone will develop inside the focus. Thus, we distinguish three circular zones. (i) An outer zone where disease severity is so low that the contribution to spore production is negligible. (ii) An intermediate zone, the productive zone, where disease level and spore production are high. In a mature focus, the width of this zone is constant. (iii) A central zone, the burnt-out zone, that no longer produces spores. Since the inner and outer boundaries of the intermediate zone increase linearly with time, total area of the productive zone also increases linearly with time. Therefore, we choose as a first approximation of the spore production by a focus of age $a$

$$
g(a)=\alpha a
$$

For reasons of comparison, we choose as an alternative description

$$
g(a)=\alpha
$$

implying a constant rate of spore production by a focus.

Spore-dispersal function. Consider the following simple description of between-field spore dispersal. Driven by wind, a spore flies at a constant velocity denoted by $v$. The flight direction makes an angle, $\theta$, with the positive $x_{1}$ axis. With a probability $\gamma$ per unit of time, the wind direction changes. The new direction, $-\pi<\theta<\pi$, is chosen at random. The spore is deposited with a probability $\mu$ per unit of time. The equation describing this dispersal process is given in appendix II. Here, we use two limiting cases of the model.

When the average flight duration, $1 / \mu$, is very small compared with the average duration of the air flow in a fixed direction, $1 / \gamma$, the spore-dispersal distribution is approximated by the exponential distribution

$$
D(\vec{x})=\frac{1}{2 \pi \sigma^{2} \sqrt{x_{1}^{2}+x_{2}^{2}}} \exp \left(-\frac{1}{\sigma} \sqrt{x_{1}^{2}+x_{2}^{2}}\right)
$$

in which $\sigma^{2}=v^{2} / \mu^{2}$ is the variance of the marginal spore-dispersal density (equation 10b).

When the average time length during which a spore is airborne, $1 / \mu$, is very large compared with the average duration of traveling in a fixed direction, $1 / \gamma$, the spore-dispersal distribution approximately follows the Bessel density (32)

$$
D(\vec{x})=\frac{1}{8 \pi \sigma^{2}} \int_{0}^{\infty} \frac{1}{\tau} \exp \left(-\tau-\frac{x_{1}^{2}+x_{2}^{2}}{\tau 8 \sigma^{2}}\right) d \tau
$$

in which $\sigma^{2}=2 v^{2} /(\gamma \mu)$ is the variance of the marginal spore-dispersal density (equation 10b). Figure 2 compares transects through the Bessel and exponential densities.

\section{RESULTS}

Epidemic spread within one cropping season. The two submodels for the spore-production function $g(a)$ and the two submodels for the spore-dispersal density $D\left(x_{1}, x_{2}\right)$ combine to give four special cases of the model. The within-season velocity of pandemic spread, $C$, and the steepness parameter of the exponential disease profile, $\lambda$, are calculated using equations 9 and 10. Table 2 summarizes the results of these straightforward but lengthy calculations. The two special cases with the Bessel distribution give explicit solutions for $C$ and $\lambda$. For the two cases with the exponential
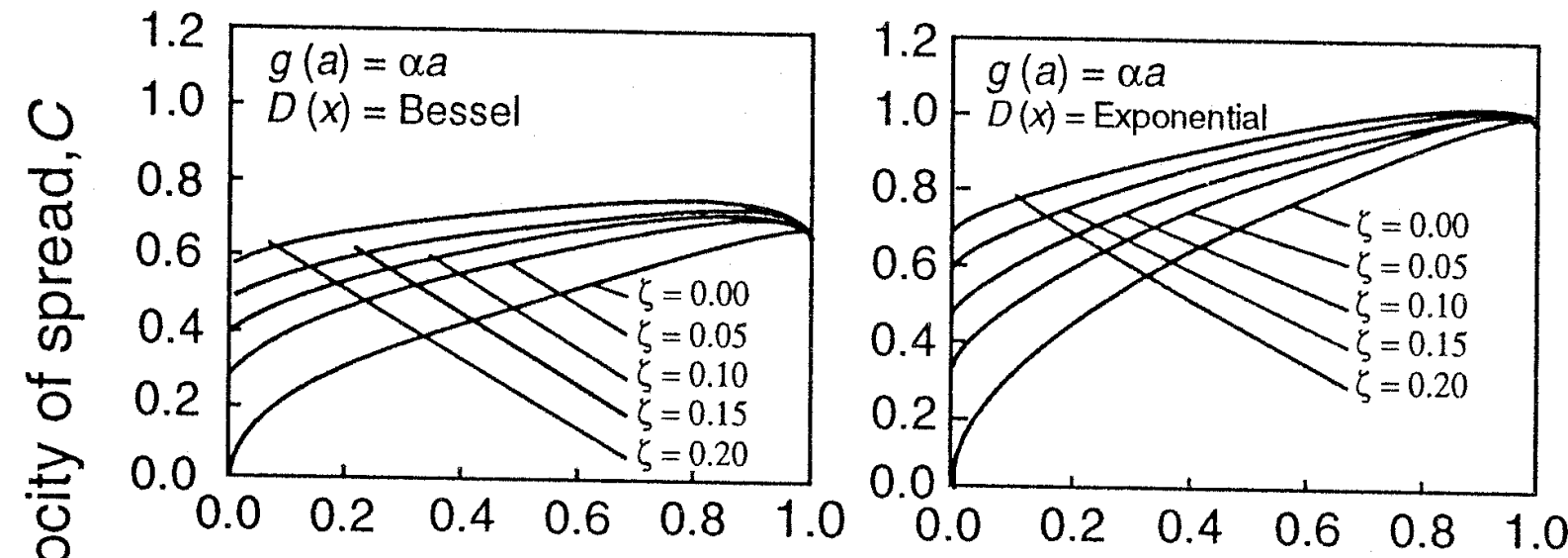

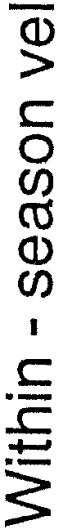
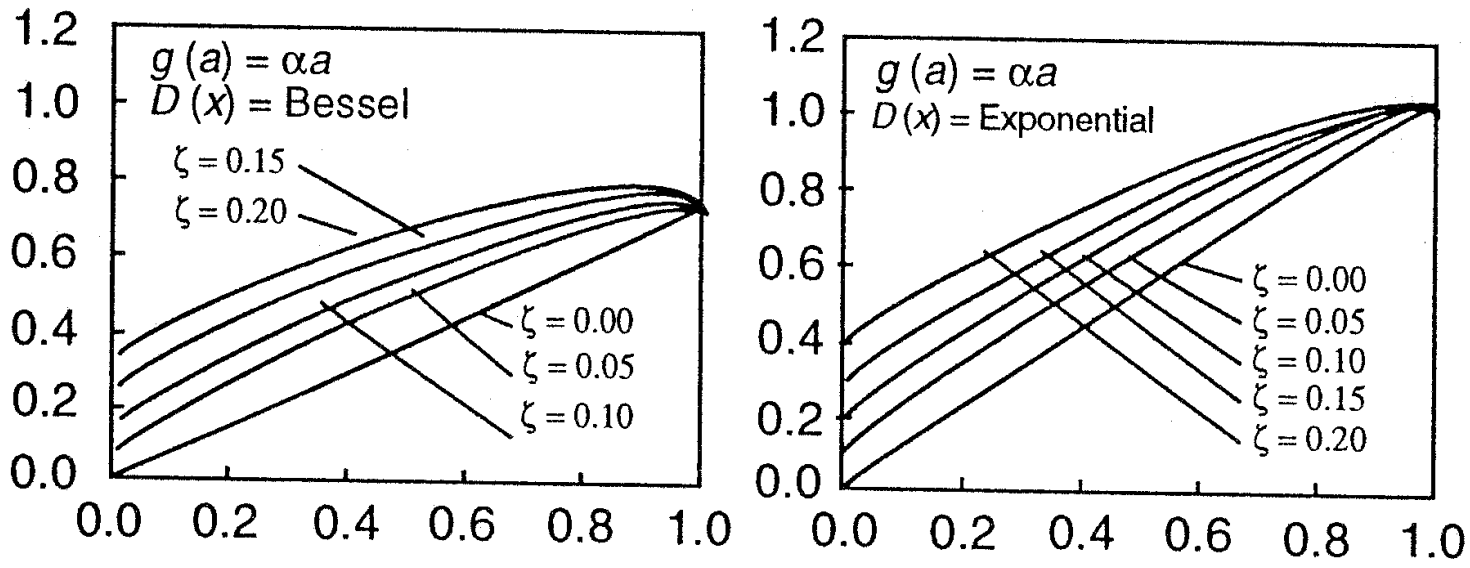

Fraction within - field spores, $\mathrm{K}$

Fig. 3. Relative within-season velocity of disease spread: $C / \sigma \alpha \psi$ when $g(a)=\alpha a$ and $C / \sigma \sqrt{ } \alpha \psi$ when $g(a)=\alpha$, as a function of the fraction of within-field spores, $\kappa$, for the four special cases studied. Parameters and functions are explained in Table 1. 
distribution, a cubic was solved numerically by a Newton-iteration subroutine (22).

Table 2 shows that in all four cases the velocity of pandemic spread, $C$, depends linearly on the standard deviation, $\sigma$, of the marginal dispersal density. This dependence, which also follows from scaling arguments, is not a property specific to the special cases, but holds (30) for any model of the form (5).

In addition, the solution always depends on two parameter groups, $K=\alpha \psi \kappa$ and $A=\zeta[(1-\kappa) / \kappa]$. The first compound parameter, $K$, can be interpreted as a measure of within-field effectiveness of the pathogen. It is composed of the overall effectiveness of the pathogen, $\alpha \psi$, and the probability that a spore stays in the same field, $\kappa . A$ is the ratio of the between-field and the withinfield effectiveness, i.e., the ratio of the number of daughter foci in distant fields and in the field of the mother focus. The dependence on $A$ is complicated. $K$ always appears in the formula for $C$ as a scaling parameter, but in different fashions depending on the precise model for spore production.

The fact that $\kappa$ appears in both $K$ and $A$ hampers the interpretation of the mathematical results in biological terms. Therefore, we shall concentrate on the dependence of $C$ on the separate parameters. The dependence of $C$ on $\alpha$ and $\psi$ is directly inherent from its dependence on $K$. For the linear spore-production function, $C$ is proportional to $\alpha \psi$; for the constant spore-production function, $C$ is proportional to the square root of $\alpha \psi$. The lesson is that good information about the dependence of the spore-production function on the age of the focus is crucial for quantitative predictions.

Next, we consider the effects of the fractions of within-field spores, $\kappa$, and of the cropping ratio, $\zeta$, on the velocity of pandemic spread, $C$. Figure 3 shows the dependence of the velocity on these two parameters for all four cases. There are only minor qualitative differences between the four cases. The velocity of pandemic spread increases with the cropping ratio to a minor extent only. A much larger effect has the fraction of within-field spores, $\kappa$. The dependence of within-season velocity of pandemic spread on $\kappa$ is remarkable. For $\kappa=0$, no spores are redeposited in the field of origin. Increasing $\kappa$ results in larger velocities, $C$. This effect holds for most values of $\kappa$ and $\zeta$, except for $\kappa$ values close to unity. At high values of $\zeta(\zeta>0.5)$, which are unrealistic, the velocities again decrease continuously with increasing $\kappa$ values.

The most surprising observation is that when $\kappa$ approaches unity, implying fewer and fewer between-field spores, the high velocity of pandemic expansion is maintained. This effect was described by Goldwasser et al. (10), who studied the velocity of population expansion of a species consisting of mobile and immobile types, with the degree of mobility determined probabilistically at birth. They also found that an extremely small number of between-field dispersers can drive a surprisingly fast population expansion. This result implies that containment measures that try to reduce the numbers of between-field spores are bound to fail in stopping or slowing down pandemic spread.

The dependence of $C$ on $\kappa$ for $\kappa$ near zero is mimicked by the dependence of $C$ on the cropping ratio $\zeta$. At low values of $\zeta$, most between-field spores fail to initiate a new focus, since they are not deposited in a target field. Yet, the epidemic speed does not approach zero. This result does not mean that the epidemic can spread without target fields, only that at low but finite cropping ratios the lack of success of spores in finding target fields becomes of minor importance relative to the growth of the epidemic within the scarce source fields to the extent of causing a discontinuity of $C$ at $\zeta=0$.

Quantitative differences are found for the within-season velocity of pandemic spread, $C$, between the Bessel and the exponential dis-

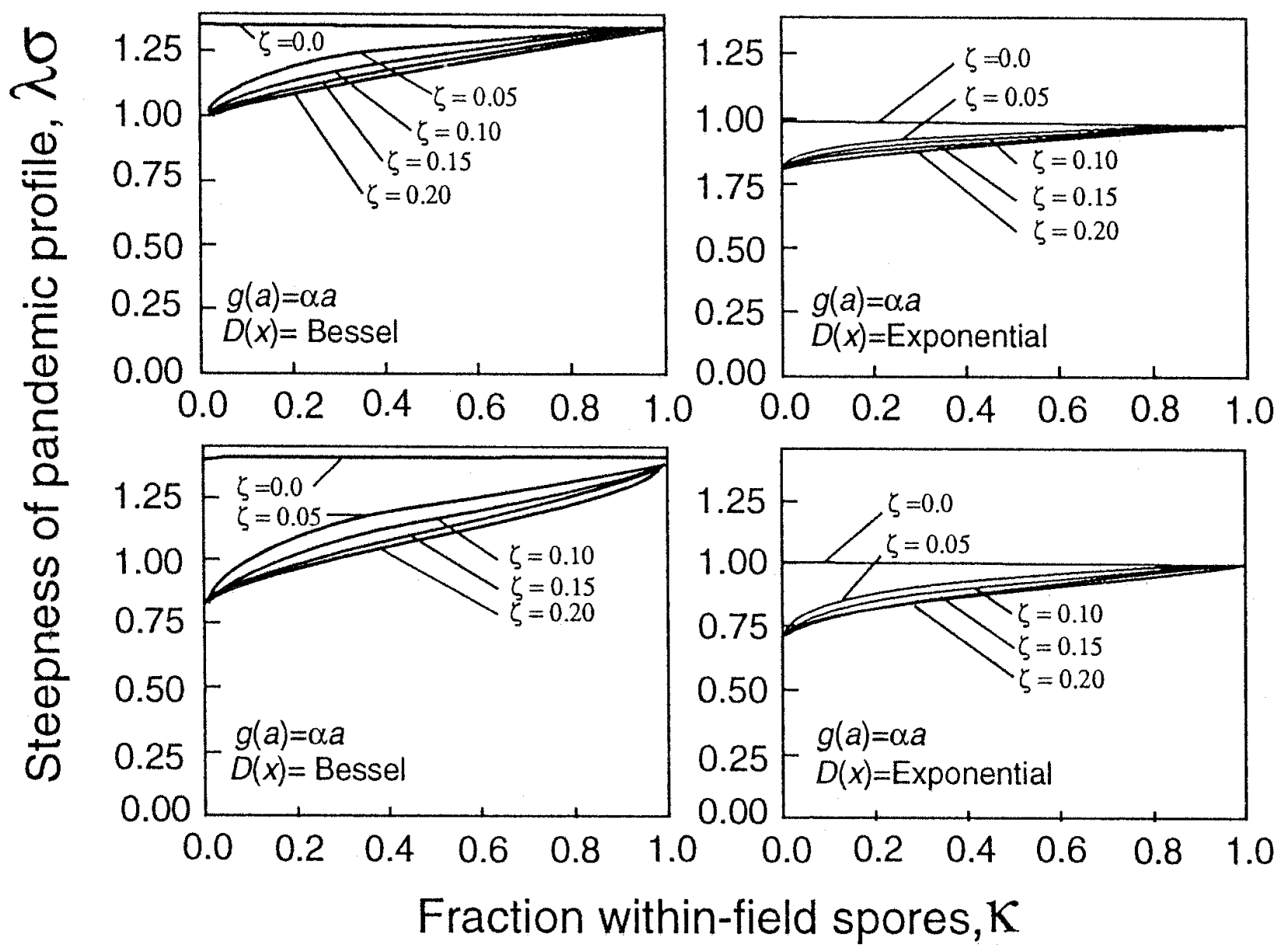

Fig. 4. Relative steepness of the pandemic disease profile, $\lambda \sigma$, as a function of the fraction of within-field spores, $\kappa$, for all four special cases studied. Parameters and functions explained in Table 1. 
persal densities; the velocities for the Bessel density being smaller than for the exponential density. This quantitative difference is more pronounced for larger values of $\kappa$. The difference is explained by the difference in the thickness of the tails of the exponential and Bessel densities (Fig. 2).

Next, we consider the measure of severity increase for a distant observer, $\lambda$. Table 2 shows that the parameters $\alpha$ and $\psi$ have no effect on this measure of severity increase of the pandemic. Clearly, the effectiveness of the disease does not affect the pandemic profile. The steepness of the disease profile is only influenced by factors related to the dispersal of the disease.

Figure 4 shows that the measure of severity increase for a distant observer, $\lambda$, slightly increases with increasing $\kappa$ for all four cases. The larger the fraction of within-field spores, the larger the rate of focus initiation in an already infected field. At the same time, the infection pressure on distant fields decreases. It is obvious that under these circumstances the steepness of the pandemic disease profile increases. Increasing the fraction of target area, $\zeta$, flattens the pandemic disease profile. This effect can be interpreted in the same sense as the effect of the fraction of within-field spores, $\kappa$. The larger the values of $\zeta$, the more foci are initiated at distant places relative to the number initiated in the source field. Changing parameter values so that the velocity of pandemic spread increases can thus steepen $(\kappa)$ or flatten $(\zeta)$ the disease profile.

Table 2 shows that the measure of severity increase usually depends on the parameter combination $A=\zeta(1-\kappa) / \kappa$. When the ratio of between-field- and within-field-deposited spores decreases, the steepness of the pandemic disease profile increases in all four cases. We conclude that the more a focus steers-up the epidemic in its own field relative to the number of daughter foci produced in dis- tant fields, the steeper the pandemic profile and thus the larger the measure of severity increase for a distant observer, as could be expected.

The rate of severity increase in a field is found from the product of velocity $C$ and steepness parameter $\lambda$. The combination of Figures 3 and 4, with $C \lambda$ on the $y$ axis, hardly alters the shape of the $C \lambda$ curves from Figure 3 . The curves only come closer to each other. Thus, the rate of severity increase in a field is hardly influenced by the cropping ratio $\zeta$. The parameter with the largest effect on the rate of severity increase is the fraction within-field spores, $\kappa$, as expected, since $\kappa$ has a large effect on both the velocity and the steepness of the pandemic profile.

Epidemic spread during successive cropping seasons. Since the within-season velocity of pandemic spread, $C$, depends linearly on $\sigma$ and $\lambda$ depends inversely on $\sigma$ (Table 2 ), we conclude from equation 15 that the polyetic velocity of pandemic spread, $V$, also increases linearly with increasing standard deviation, $\sigma$, of the marginal dispersal density, as might be expected.

Equation 14 shows that the push-back distance, $\Delta h$, during the off-season is proportional to $1 / \lambda$, so that a steep pandemic disease profile is pushed back over a short distance and a flat profile is pushed back over a large distance. The equation further shows that the push-back distance is proportional to the logarithm of the fraction, $\varepsilon$, of the foci established after the crop-free period.

In the previous section, the within-season velocity of pandemic spread, $C$, was analyzed. We concluded that there was no qualitative difference between the four cases analyzed. Calculations indicate that this qualitative independence is also found in the polyetic velocity of pandemic spread (data not shown). Therefore, we restrict our attention to the case of a Bessel dispersal density, equation 19,

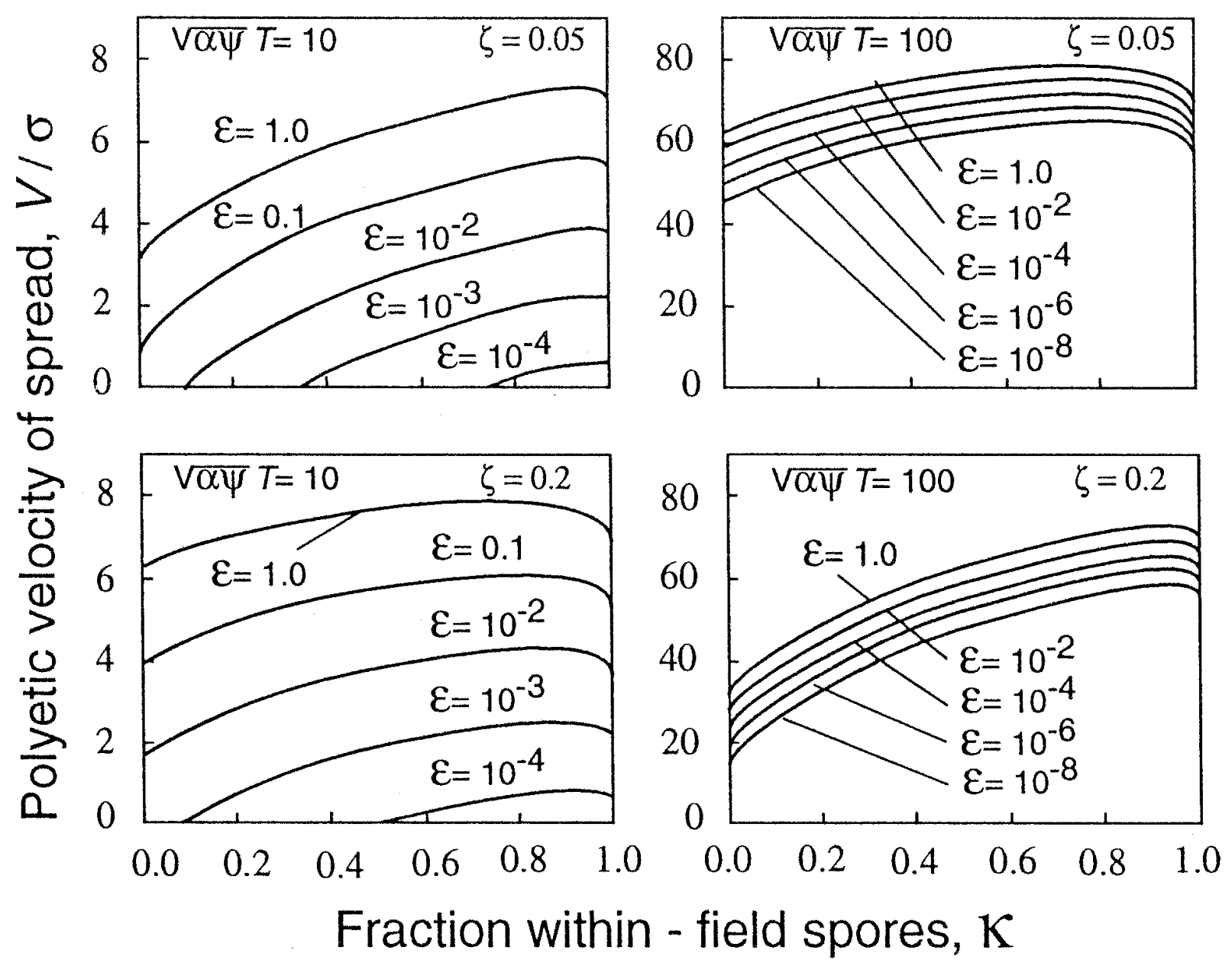

Fig. 5. Relative polyetic or between-season velocity of pandemic spread, $V / \sigma$, as a function of the fraction of within-field spores, $\kappa$, at various values of $\varepsilon$. Parameters and functions explained in Table 1. 
and a linear increase of spore production with age, equation 16 . In this case,

$$
V=\frac{\sigma}{\sqrt{2}} \frac{\sqrt{\alpha \psi} \sqrt{\kappa} T \sqrt{1+A+\sqrt{A^{2}+A}}-\ln \left(\frac{1}{\varepsilon}\right)}{\sqrt{1+A-\sqrt{A^{2}+A}}}
$$

in which

$$
A=\zeta \frac{1-\kappa}{\kappa}
$$

We will first discuss parameter dependence of the push-back distance. The survival of foci, $\varepsilon$, is, as expected, the major determinant of push-back distance. The push-back distance is independent of the parameter combination, $\alpha \psi$, interpreted as a measure of effectiveness of the disease. Push-back distance depends linearly on the standard deviation of the marginal spore-dispersal density. Next, consider the term $B=\sqrt{ }\left[1+A-\sqrt{ }\left(A^{2}+A\right)\right]$, in which $A$ is given in equation 21. $B$ decreases from $B=1$ for $A=0$ and $B=$ 0.765 for $A=1$ to $B=0.707$ for $A \rightarrow \infty$. Since the factor $(1-\kappa) / \kappa$ ranges from $0(\kappa=1)$ to $\infty(\kappa=0)$, we conclude that the pushback distance depends on $\kappa$ to a minor extent only. For the cropping ratio, $\zeta$, ranging from 0 to 1 , we also conclude that the pushback distance hardly depends on $\zeta$.

Next, we turn to the polyetic velocity of spread, $V$. From Figure 5 , we conclude that decreasing survival of foci, $\varepsilon$, decreases rates of spread. This, of course, only through its effect on the push-back distance. Figure 6, however, shows that there is a large difference in the relative effect of $\varepsilon$ on $V$ for different values of the effectiveness, $\alpha \psi$. The survival of foci has a large influence on the polyetic velocity of pandemic spread, $V$, when the disease has a small $T \sqrt{ } \alpha \psi$ value. Measures to reduce the velocity of disease spread by reducing survival during the crop-free period are thus successful only for diseases with small $T \sqrt{ } \alpha \psi$ values.

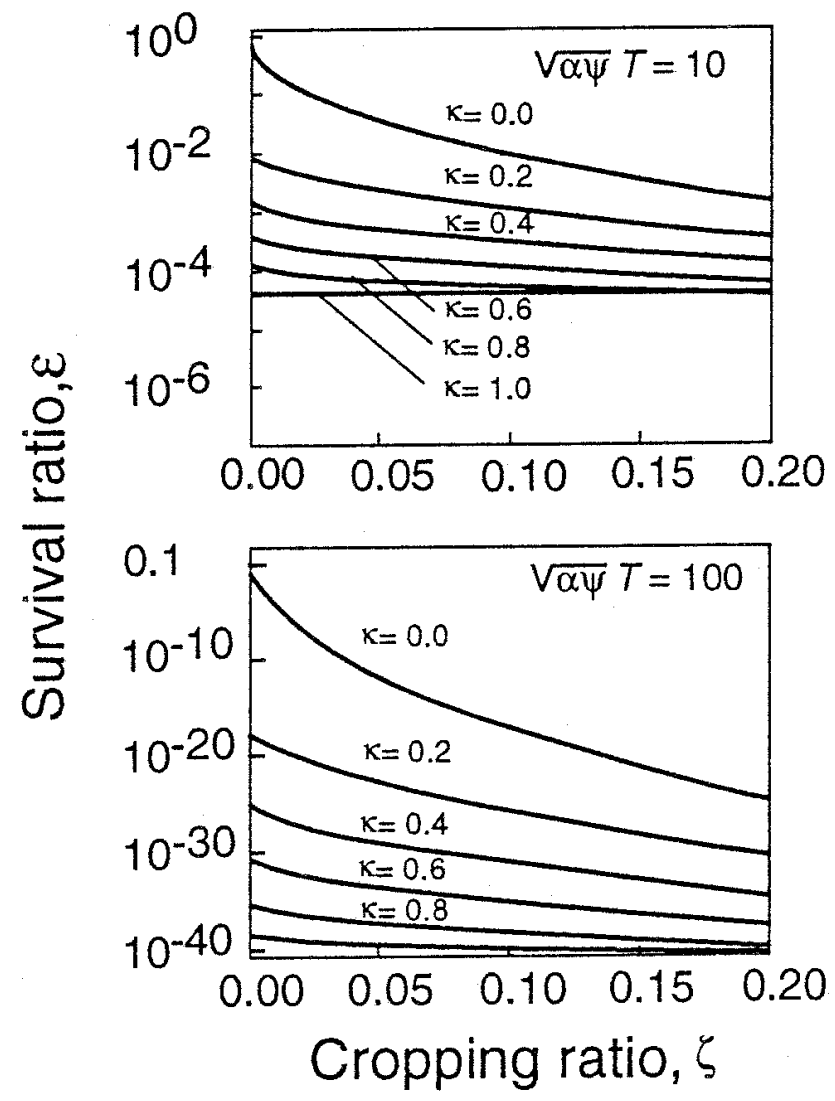

Fig. 6. Threshold survival ratio, $\varepsilon$, below which the disease dies out, plotted against the cropping ratio, $\zeta$, for different values of the fraction of within-field spores, $\kappa$.
Figure 5 shows the polyetic velocity of pandemic spread, $V$, as a function of the fraction of within-field spores, $\kappa$, for several values of the other parameters. Again, the polyetic velocity of pandemic spread increases with increasing effectiveness, $\sqrt{ } \alpha \psi$, of the disease.

The rate $V$ increases with an increasing fraction of within-field spores, $\kappa$, except for values of $\kappa$ close to unity. This remarkable relation was also found for the within-season rate, $C$, and the same explanation holds.

Last but not least, the cropping ratio, $\zeta$, has, compared with other parameters, a minor effect on the polyetic velocity $V$. This is due to the fact that it has a minor effect on both the push-back distance and on the within-season velocity of spread. Note that this last result immediately implies that our results will change to a minor extent only when crop fields are not distributed homogeneously over the continent.

Decreasing survival of foci during the crop-free period can reduce the rate of pandemic spread to zero, implying complete control of the disease. Setting $V$ equal to zero in equation 20 , we find the threshold value of the survival fraction, $\varepsilon$, in which the rate of pandemic spread becomes zero. Complete control of the disease is attained when

$$
\varepsilon \leq \exp \left(-\sqrt{\alpha \psi} \sqrt{\kappa} T \sqrt{1+A+\sqrt{A^{2}+A}}\right)
$$

Figure 6 shows the off-season survival ratio of foci, $\varepsilon$, as a function of the cropping ratio, $\zeta$. The threshold value of $\varepsilon$ depends to a minor extent on $\zeta$. Only for $\kappa$ values close to zero, the necessary $\varepsilon$ decreases considerably with increasing $\zeta$. Figure 6 confirms that prevention of the pandemic by reducing $\varepsilon$ only seems an interesting control strategy for pathogens with low effectiveness; i.e., diseases with low $\alpha \psi$.

The method introduced to derive the polyetic velocity of pandemic spread shows that the measure of severity increase for a distant observer, $\lambda$, is the same as for within-season pandemic spread. We conclude that severity increase is not influenced by parameters of between-season survival of the disease. The discussion on parameter dependence of the severity increase in the withinseason spread applies also to the polyetic spread. Combining Figures 4 and 5 we can, as for within-season spread, conclude that the shape of the $V \lambda$ curves does not change much from Figure 5, and again, the discussion on severity increase for within-season spread applies to polyetic spread as well.

Generalizations. Nonhomogeneous physical environment. The model equations 4 make two essential assumptions about the physical environment. First, the target fields are assumed to be homogeneously distributed through space. Second, we assumed that dispersal is rotationally symmetric and not influenced by the position of the source field on the continent. In real-life crop fields, these assumptions will not be met. Crop fields usually show a high degree of clustering. When tobacco blue mold, for example, invaded western Europe, tobacco production occurred in limited areas, whereas most of western Europe was a nontarget area (21). In addition, spore dispersal from a field will vary according to the field's position: on the coast, inland, or in the mountains. Inhomogeneous crop field distributions will not influence the velocity of pandemic spread to a great extent. When the spatial scale of the inhomogeneity of the field distribution is smaller than the spatial extent of the spore-dispersal density, measured by $\sigma$, the model predictions are an accurate approximation due to averaging. Moreover, in the previous section, we concluded that the cropping ration, $\zeta$, has, compared with other parameters, a minor effect on velocities of spread both for within-season as for polyetic spread. From this, we may expect that the presence of large-scale inhomogeneities in cropping ratio have only a minor effect on the pattern of epidemic spread.

In circumstances in which one would like to study nonhomogeneity, the assumptions of the model are easily relaxed. First, define 
the cropping ratio to be dependent on the position on the continent and write for the cropping ratio $\zeta(\vec{x})$. Second, use the general description $D(\vec{x}, \vec{\varphi})$ for the probability that a spore produced at $\vec{\varphi}$ is deposited at $\vec{x}$. Substituting the position-dependent cropping ratio and dispersal density into model equations 4 yields the generalized model

$$
\begin{gathered}
b(t, \vec{x})=\psi \kappa \int_{0}^{t} b(t-a, \vec{x}) g(a) d a+\psi v(t, \vec{x}) \\
v(t, \vec{x})=\zeta(\vec{x})(1-\kappa) \int_{0}^{t} \int_{-\infty}^{\infty} \int_{-\infty}^{\infty} b(t-a, \vec{\varphi}) g(a) D(\vec{x}, \vec{\varphi}) d \varphi_{1} d \varphi_{2} d a
\end{gathered}
$$

To account for geographical elements such as large bodies of water, jutting peninsulas, and continental boundaries, the cropping ratio is set to zero for the positions where no crop can occur. In the example given to develop $D(\vec{x})$, prevailing wind directions can be added by relaxing the assumption that after directional change the new direction is chosen randomly from all possible angles (20).

For nonrotationally symmetric dispersal densities, the pandemic velocities can be calculated analytically as long as $D$ is translationally invariant $(16,28)$. During a growing season, the wind may change unpredictably and blow in any direction with highly variable directional frequency, as is common in Europe. Since the foci established furthest away matter most for the pandemic velocity, the effect of variation in directional frequency of the wind may even out and rotational symmetry may be maintained to a fair degree. European data (11) seem to confirm this point, which merits more attention.

The generalized model (23) does not allow traveling-wave solutions of the type studied in this paper. Some analytical results on a diffusion-type model have been published $(25,26)$. A further study of the spatial spread in heterogeneous environments is urgently needed but, probably, analytical solutions of equation 23 will have to give way to numerical approaches $(35,38)$. Realistic submodels for $D(\vec{x}, \vec{\varphi})$ can be developed using the work of Aylor (2) as a guideline.

Severity dependence. Model equations 4 assume that a fraction $\psi$ of the spores deposited in a target field always produce a new focus. This is no longer true when disease severity in the field, or the number of foci in the field, becomes large. Moreover, equation 1 assumes that the focal rate of spore production, $g(a)$, is not influenced by the number of foci in the field. Again, this is no longer true when the foci begin to overlap. Both types of severity dependence can be incorporated into model equations 4 . Denote by $O(a)$, the area of a focus of focal age $a$. The sum of the focal areas in a field at position $\vec{x}, M(t, \vec{x})$, is calculated from

$$
M(t, \vec{x})=\int_{0}^{t} O(a) b(t-a, \vec{x}) d a
$$

The field area is denoted by $\Omega$. From the first term of the Poisson distribution, we now find the probability that a spore deposited in a target field at position $\vec{x}$ is not deposited in a focus, $P(t, \vec{x})$,

$$
P(t, \vec{x})=1-\frac{1}{\Omega} \int_{0}^{t} O(a) b(t-a, \vec{x}) d a
$$

Multiplying $P$ with the probability for a spore to start a new focus, $\psi$, we have the probability that a spore deposited at time $t$ in a field at position $\vec{x}$ starts a new focus. To account for focal overlap in the rate of spore production, we use a similar approach. In choosing submodels for $g(a)$, we defined the burntout zone of a focus as the central zone that no longer produces spores. In the severity-dependent situation in which foci overlap, the area of overlap will be close to burnt-out. Thus, $g(a)$ has to be multiplied by the fraction of the foci that do not overlap with other foci, $H(t, \vec{x})$.
Substituting the severity-dependent processes in model equations 4 , we find

$$
\begin{aligned}
& b(t, \vec{x})=\psi\left[1-\frac{1}{\Omega} \int_{0}^{t} O(a) b(t-a, \vec{x}) d a\right]\left[\kappa \int_{0}^{t} b(t-a, \vec{x}) g(a) H(t, \vec{x}) d a+\mathrm{v}(t, \vec{x})\right] \\
& \nu(t, \vec{x})=\zeta(1-\kappa) \iint_{0-\infty}^{t} \int_{-\infty}^{\infty} \int^{\infty} b(t-a, \vec{\varphi}) g(a) H(t, \vec{x}) D(|\vec{x}-\vec{\varphi}|) d \varphi_{1} d \varphi_{2} d a
\end{aligned}
$$

Several possible models can be developed for $H(t, \vec{x})$. A simple first approximation could be to use $H(t, \vec{x})=P(t, \vec{x})$ in equation 25 . Another possible approach is described by Shigesada and Kawasaki (24). An important question is whether this severity dependence influences the velocity of pandemic spread. At the continental scale, the severity dependence will come into play at a certain position some time after the front of the pandemic has passed this position. Several related models $(6,13,28,34)$ for the spatial spread of populations show that the velocity of population spread is exactly the same in density-dependent and density-independent situations. The mathematical proofs have a biologically appealing result. A population spreads through space by intruding into areas where it was absent, the population wave being dragged forward by its tail. Far in the front of the wave, the epidemic does not experience any density-dependent effects, since population densities are low. Since the mathematical evidence $(6,13,28$, 34) accounts for rather general classes of models, we can safely assume, by analogy, that the velocities of pandemic spread of the severity-dependent and the severity-independent model are similar, although a proof for the current model does not exist. An analytical solution for the case that foci overlap and the field 'fills up' is not yet available.

Dispersal models with nonexponential tails. As stated above, the calculation of the velocity of pandemic spread using equations 9 and 10 is possible only when the tails of the between-field-dispersal density are exponentially bounded. For nonexponentially bounded tails, model equations 4 still applies. Ferrandino (7) developed a spore-dispersal density with nonexponentially bounded tails based on detailed considerations of wind velocity and turbulence. This dispersal density was incorporated in an epidemic model. Numerical solutions of the model suggested that the velocity of epidemic spread increased continually. Ferrandino called such waves 'dispersive epidemic waves.' In a recent paper (14), the existence of such dispersive waves was proven in simple analytically tractable models of population invasions and methods were developed to approximate the velocity of the dispersive wave as a function of time since the introduction of the species. It would be worthwhile to apply their methods to the current model, but this will be a major mathematical task. Efficient methods to simulate epidemics with nonexponential tails are available (23).

\section{DISCUSSION}

This paper develops a framework for modeling the continental spread of focal plant disease. Methods to calculate the velocity of pandemic spread were developed both for within-season spread and for polyetic spread (during successive cropping seasons). To facilitate the study of the model by analytical methods, rather than by computer simulation, the information on the mechanisms underlying pandemic spread was stripped to bare essentials. Thus, several potentially important aspects are not yet incorporated in the current analysis. Subsequently, several generalizations are discussed that account for these aspects.

The general model equations 4 can be adapted to describe a particular disease by an appropriate choice of $g(a)$ and $D(\vec{x})$. Detailed and realistic descriptions of the spore-production function, $g(a)$, and the dispersal distribution, $D(\vec{x})$, will almost certainly be parameter rich. Parameter dependence of the velocity of pandemic spread is difficult to study in such parameter-rich mod- 
els. Therefore, we restricted this first attempt at modeling pandemic spread to simple parameter-sparse submodels. Future research should introduce more detailed models for $g(a)$ and $D(\vec{x})$ and include the development of models for between-field dispersal (2).

Simple analytically tractable models, like the one discussed in this paper, are useful tools to gain qualitative insight in the dependence of the velocity of pandemic spread on the underlying processes. Sometimes, such models can be put to work in real-life situations. A focus expansion model was validated using laboratory and field data (29,31-33). It will be difficult, if possible at all, to perform similar validation studies using the current model.

We think that the current model can best be used as a means to compare pandemics rather than as a stimulus to measure all input parameters experimentally. Comparisons can be made, for example, between pandemics of a plant disease that invaded at various places in the world with different cropping ratios or different climatic conditions.

\section{APPENDIXES}

I. Reformulating the model. In this appendix, we show that the model equations 4 are equivalent to equations 5 and 6 . To this end, we use the technique of Laplace transformation. An introduction to Laplace transformation and its application are described by Churchill (3). The Laplace transform of a function $f(t)$ is defined as

$$
L[f(t)]:=\bar{f}(s):=\int_{0}^{\infty} e^{-s t} f(t) d t
$$

Laplace transforms of a large number of functions are described by Abramowitz and Stegun (1). Laplace transforms are particularly useful in solving linear differential and integral equations, since these equations are transformed into algebraic expressions. As an example, the integral equation

$$
b(t)=\int_{0}^{t} a b(t-a) d a+t
$$

seems unsolvable at first sight. It has Laplace transform

$$
\bar{b}(s)=\bar{b}(s) \frac{1}{s^{2}}+\frac{1}{s^{2}} \Leftrightarrow \bar{b}(s)=\frac{1}{s^{2}-1}
$$

From the tables by Abramowitz and Stegun (1), we find

$$
L[\sinh (t)]=\frac{1}{s^{2}-1}
$$

and the solution of the integral equation I.2 thus is $b(t)=\sinh (t)$. We apply these methods to our model. Taking Laplace transforms of the model equations 4 with respect to $t$, we find

$$
\begin{gathered}
\bar{b}(s, \vec{x})=\psi \bar{v}(s, \vec{x})+\psi \kappa \bar{b}(s, \vec{x}) \bar{g}(s) \\
\bar{v}(s, \vec{x})=\zeta(1-\kappa) \int_{-\infty}^{\infty} \int_{-\infty}^{\infty} \bar{b}(s, \vec{\varphi}) \bar{g}(s) D(|\vec{x}-\vec{\varphi}|) d \varphi_{1} d \varphi_{2}
\end{gathered}
$$

in which

$$
\bar{b}(s, \vec{x})=\int_{0}^{\infty} e^{-s t} b(t, \vec{x}) d t ; \bar{v}(s, \vec{x})=\int_{0}^{\infty} e^{-s t} v(t, \vec{x}) d t ; \bar{g}(s)=\int_{0}^{\infty} e^{-s a} g(a) d a \text { (I.6) }
$$

Solving for $b(s, \vec{x})$ in the first equation of I.5, we find

$$
\bar{b}(s, \vec{x})=\frac{\psi \bar{v}(s, \vec{x})}{1-\psi \kappa \bar{g}(s)}=\psi \bar{v}(s, \vec{x}) \sum_{i=1}^{\infty}[\psi \kappa \bar{g}(s)]^{i-1}=\frac{\bar{v}(s, \vec{x})}{\kappa \bar{g}(s)} \sum_{i=1}^{\infty}[\psi \kappa \bar{g}(s)]^{i} \text { (I.7) }
$$

Substituting the second equation of I.5, we find

$$
\bar{b}(s, \vec{x})=\zeta(1-\kappa) \int_{-\infty}^{\infty} \int_{-\infty}^{\infty} \frac{1}{\kappa} \bar{b}(s, \vec{\varphi}) \sum_{i=1}^{\infty}[\psi \kappa \bar{g}(s)]^{i} D(|\vec{x}-\vec{\varphi}|) d \varphi_{1} d \varphi_{2}
$$

and taking inverse Laplace transforms finally yields equations 5 and 6 .

II. The spore dispersal distribution. A detailed account of the type of model formulation used in this appendix is given by Othmer et al. (20). We will use their method to derive the spore-dispersal distribution.

A spore is dispersed by wind. It travels with velocity $v_{1}$ in the $x_{1}$ direction and with velocity $v_{2}$ in the $x_{2}$ direction. With a probability $\gamma$ per unit of time, the wind changes its direction. The new winddirection vector, $\left(v_{1}, v_{2}\right)$, is chosen randomly from a distribution $Q\left(v_{1}, v_{2}\right)$. The spore is deposited on the surface with probability $\mu$ per time unit.

Denote by $p\left(t, x_{1}, x_{2}, v_{1}, v_{2}\right)$ the probability density of a spore to be at time $t$ at position $\left(x_{1}, x_{2}\right)$ and traveling with velocity vector $\left(v_{1}, v_{2}\right)$. The spore-dispersal process is governed by the model equation

$$
\begin{aligned}
& \frac{\partial p\left(t, x_{1}, x_{2}, v_{1}, v_{2}\right)}{\partial t}= \\
& -v_{1} \frac{\partial p}{\partial x_{1}}-v_{2} \frac{\partial p}{\partial x_{2}}-\gamma p-\mu p+\gamma Q\left(v_{1}, v_{2}\right) \int_{-\infty}^{\infty} \int_{-\infty}^{\infty} p\left(t, x_{1}, x_{2}, v_{1}^{\prime}, v_{2}^{\prime}\right) d v_{1}^{\prime} d v_{2}^{\prime}
\end{aligned}
$$

Taking Laplace transforms with respect to time $s$ and position $\left(z_{1}, z_{2}\right)$, we find

$$
s \bar{p}\left(s, \lambda_{1}, \lambda_{2}, v_{1}, v_{2}\right)-Q\left(v_{1}, v_{2}\right)=-\sum_{i} v_{i} \lambda_{i} \bar{p}-(\gamma+\mu) \bar{p}+Q\left(v_{1}, v_{2}\right) \gamma \bar{P}
$$

with

$$
P\left(t, x_{1}, x_{2}\right)=\int p\left(t, x_{1}, x_{2}, v_{1}^{\prime}, v_{2}^{\prime}\right) d v_{1}^{\prime} d v_{2}^{\prime}
$$

The deposition kernel is then given by

$$
\mu \bar{P}=\frac{\mu F\left(s, \lambda_{1}, \lambda_{2}\right)}{1-\gamma F\left(s, \lambda_{1}, \lambda_{2}\right)}
$$

in which

$$
F\left(s, \lambda_{1}, \lambda_{2}\right)=\iint \frac{Q\left(v_{1}, v_{2}\right)}{\gamma+\mu+s+\sum \lambda_{i} v_{i}} d v_{1} d v_{2}
$$

Assuming $Q$ to be homogeneously distributed on the circle with radius $\mathrm{v}$, we have

$$
F\left(s, \lambda_{1}, \lambda_{2}\right)=\frac{1}{2 \pi} \int_{0}^{2 \pi} \frac{d \theta}{\gamma+\mu+s+v\left[\lambda_{1} \cos (\theta)+\lambda_{2} \sin (\theta)\right]}
$$

Now, to calculate the Laplace transform of the marginal dispersal density, put $\lambda_{1}=\lambda$ and $\lambda_{2}=0$, substitute II. 6 into II. 4 to arrive at

$$
\mu \bar{P}=\frac{1}{\sqrt{\left(\frac{\gamma+\mu+s}{\mu}\right)^{2}-\left(\frac{v \lambda}{\mu}\right)^{2}}-\frac{\gamma}{\mu}}
$$

The two special cases are found as follows.

Taking the limit for $\gamma \downarrow 0, \mu \rightarrow \infty$, and $\nu \rightarrow \infty$ such that $\nu / \mu$ remains constant, we find

$$
\mu \bar{P}=\frac{1}{\sqrt{1-\sigma^{2} \lambda^{2}}}
$$

in which $\sigma^{2}=v^{2} / \mu^{2}$, which is the Laplace transform of the marginal density of the rotationally symmetric exponential density (equation 18). 
Taking the limit for $\gamma \rightarrow \infty, \mu \rightarrow \infty$, and $v \rightarrow \infty$ such that $v^{2} /(\gamma \mu)$ remains constant, we find

$$
\mu \bar{P}=\frac{1}{1-1 / 2 \sigma^{2} \lambda^{2}}
$$

in which $\sigma^{2}=v^{2} / \gamma \mu$, which is the Laplace transform of the marginal density of the Bessel density (equation 19).

\section{LITERATURE CITED}

1. Abramowitz, M., and Stegun, I. A. 1965. Handbook of Mathematical Functions. Dover Publications Inc., New York.

2. Aylor, D. 1986. A framework for examining inter-regional aerial transport of fungal spores. Agric. For. Meteorol. 38:263-288.

3. Churchill, R. V. 1972. Operational Mathematics. McGraw-Hill Kogakusha, Tokyo.

4. Cooke, M. C. 1891. Tobacco disease. Gard. Chron. 9:173.

5. Diekmann, O. 1978. Thresholds and travelling waves for the geographical spread of infection. J. Math. Biol. 6:109-130.

6. Diekmann, O. 1979. Run for your life. J. Differ. Equations 33:58-73.

7. Ferrandino, F. J. 1993. Dispersive epidemic waves: I. Focus expansion within a linear planting. Phytopathology 83:795-802.

8. Fisher, R. A. 1937. The wave of advance of advantageous genes. Ann. Eugenet. 7:355-369.

9. Gäumann, G. A. 1946. Pflanzliche Infektionslehre. Birkhäuser, Basel.

10. Goldwasser, L., Cook, J., and Silverman, E. D. 1994. The effect of variability on metapopulation dynamics and rates of invasion. Ecology 75: 40-47.

11. Heesterbeek, J. A. P., and Zadoks, J. C. 1987. Modelling pandemics of quarantine pests and diseases: Problems and perspectives. Crop Prot. 6: 211-221.

12. Kendall, D. G. 1965. Mathematical models of the spread of infection. Pages 213-224 in: Mathematics and Computer Sciences in Biology and Medicine. Medical Research Council, London.

13. Kolmogorov, A., Petrovsky, I., and Piscounov, N. 1937. Etude de l'équation de la diffusion avec croissance de la quantité de matière et son application à un problème biologique. Mosc. Univ. Bull. Math. 1:1-25.

14. Kot, M., Lewis, M. A., and van den Driessche, P. 1996. Dispersal data and the spread of invading organisms. Ecology 77:2027-2042.

15. McGregor, R. C. 1978. People placed pathogens: The emigrant pests. Pages 383-396 in: Plant Disease. An Advanced Treatise, Vol. 2. J. G. Horsfall and E. B. Cowling, eds. Academic Press, New York.

16. Metz, J. A. J., and van den Bosch, F. 1995. Velocities of epidemic spread. Pages 150-186 in: Epidemic Models: Their Structure and Relation to Data. D. Mollison, ed. Cambridge University Press, Cambridge, United Kingdom.

17. Minogue, K. P., and Fry, W. E. 1983. Models for the spread of disease: Model description. Phytopathology 73:1168-1173.

18. Minogue, K. P., and Fry, W. E. 1983. Models for the spread of plant disease: Some experimental results. Phytopathology 73:1173-1176.
19. Mollison, D. 1991. Dependence of epidemic and population velocities on basic parameters. Math. Biosci. 107:255-287.

20. Othmer, H. G., Dunbar, S. R., and Alt, W. 1988. Models of dispersal in biological systems. J. Math. Biol. 26:263-298.

21. Populer, C. 1964. Le comportement des épidémies de mildiou du tabac, Peronospora tabacina. 1. La situation en Europe. Bull. Inst. Agron. Stn. Rech. Gembloux 32:339-378.

22. Press, W. H., Flannery, B. P., Teukolsky, S. A., and Vetterling, W. T. 1992. Numerical Recipes in PASCAL: The Art of Scientific Computing. Cambridge University Press, New York.

23. Shaw, M. W. 1995. Simulation of population expansion and spatial pattern when individual dispersal distributions do not decline exponentially with distance. Proc. R. Soc. Lond. B 259:243-248.

24. Shigesada, N., and Kawasaki, K. 1997. Biological Invasions: Theory and Practice. Oxford Series in Ecology and Evolution. Oxford University Press, New York.

25. Shigesada, N., Kawasaki, K., and Teramoto, E. 1986. Travelling periodic waves in heterogeneous environments. Theor. Popul. Biol. 30:143-160.

26. Shigesada, N., Kawasaki, K., and Teramoto, E. 1987. The speed of travelling frontal waves in heterogeneous environments. Pages 88-97 in: Mathematical Topics in Population Biology, Morphogenesis and Neurosciences. E. Teramoto and M. Yamaguti, eds. Lecture Notes in Biomathematics 71. Springer, Berlin.

27. Skellam, J. G. 1951. Random dispersal in theoretical populations. Biometrica 38:196-218.

28. Thieme, H. R. 1977. A model for the spread of an epidemic. J. Math. Biol. 4:337-351.

29. van den Bosch, F., Frinking, H. D., Metz, J. A. J., and Zadoks, J. C. 1988. Focus expansion in plant disease. III: Two experimental examples. Phytopathology 78:919-925.

30. van den Bosch, F., Metz, J. A. J., and Diekmann, O. 1990. The velocity of population expansion. J. Math. Biol. 28:529-565.

31. van den Bosch, F., Verhaar, M. A., Buiel, A. A. M., Hoogkamer, W., and Zadoks, J. C. 1990. Focus expansion in plant disease. IV: Expansion rates in mixtures of resistant and susceptible hosts. Phytopathology 80:598-602.

32. van den Bosch, F., Zadoks, J. C., and Metz, J. A. J. 1988. Focus expansion in plant disease. I: The constant rate of focus expansion. Phytopathology 78:54-58.

33. van den Bosch, F., Zadoks, J. C., and Metz, J. A. J. 1988. Focus expansion in plant disease. II: Realistic parameter-sparse models. Phytopathology 78:59-64.

34. Weinberger, H. F. 1982. Long-time behavior of a class of biological models. SIAM (Soc. Ind. Appl. Math.) J. Math. Anal. 13:353-396.

35. Zadoks, J. C., and Kampmeijer, P. 1977. The role of crop populations and their deployment, illustrated by means of a simulator, EPIMUL76. Ann. N.Y. Acad. Sci. 287:164-190.

36. Zadoks, J. C., and Schein, R. D. 1979. Epidemiology and plant disease management. Oxford University Press, New York.

37. Zadoks, J. C., and van den Bosch, F. 1994. On the spread of plant disease: A theory on foci. Annu. Rev. Phytopathol. 32:503-521.

38. Zawolek, M. W., and Zadoks, J. C. 1989. A physical theory of focus development in plant disease. Wageningen Agricultural University Papers 89-3. 